\title{
RHIC Run-away Type Pressure Rise
}

S.Y. Zhang

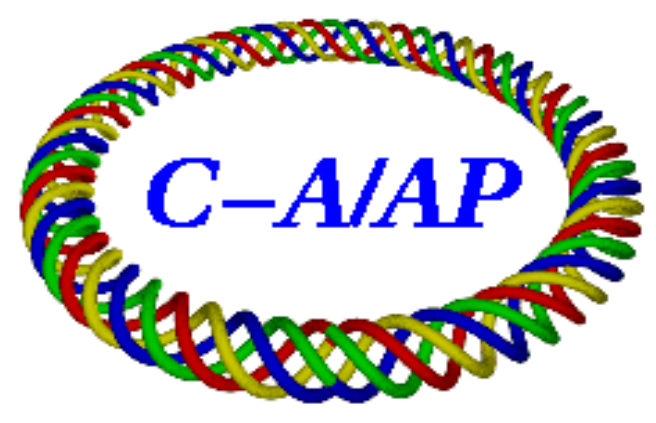

Collider-Accelerator Department Brookhaven National Laboratory Upton, NY 11973 


\title{
RHIC Run-away Type Pressure Rise
}

\author{
S.Y. Zhang
}

\section{Author's note}

This report was drafted in later 2002, and the abstract was submitted to PAC03. Since the run-away type pressure rise became less concerned in Run 2003, the report was left unfinished. A recent work [1] reminded the author that many related issues remain unsettled for the RHIC pressure rise. By completing this note, parameters regarding to the run-away type pressure rise are presented for discussion, and questions are listed for further investigation.

\section{Introduction}

The run-away type pressure rise was frequently observed in RHIC Au-Au run 2002, mostly at IR12, and always associated with beam loss. In d-Au run 2003 and Au-Au run 2004, only limited run-away type pressure rise was observed. The locations were at Yo1 and Yi2 in run 2003, and at Yo4 and Bi8 in run 2004. All run-away type pressure rise occurred in gold beam runs, but in $\mathrm{Cu}-\mathrm{Cu}$ run 2005, the pressure rise at IP4 of the fill 5906 looks like a run-away type. The concern of the run-away type pressure rise in terms of operation and intensity limit has reduced, however, the related issues remain relevant to better understanding of the RHIC pressure rise problem.

In this article, we discuss 3 possible mechanisms, which may lead to runaway type pressure rise.

1. Ion desorption induced pressure instability. This vacuum instability was observed at the CERN ISR. The beam gas ionization created ions were pushed to the wall by beam potential, and the increased pressure in turn enhanced the beam gas ionization. In the RHIC, in addition to the beam gas ionization, the electron cloud creates more ions. At 
the low energy of cloud electrons, the ionization cross section could be large. Nevertheless, for RHIC the most important factor in the ion desorption induced pressure instability is probably the energy of the ions hitting the chamber wall. Given the RHIC beam intensity, this energy is around $10 \mathrm{eV}$, which is below the threshold of sputtering. Unless some unknown factors are revealed, the ion desorption is unlikely to cause run-away type pressure rise at RHIC.

2. Beam loss induced pressure instability. This vacuum instability might have been observed at GSI. For low energy heavy ion accelerators, the beam capture, or stripping, cross sections are large. The lost beam ions may create large amount of molecules by scraping on the wall, and the increased pressure rise causes more beam loss. In the RHIC, the dominant beam loss mechanism due to gas is probably from the nuclear collision. Given the pumping capability and the RHIC beam intensity, larger than $10^{9}$ ion desorption rate is needed to create a runaway pressure rise. Perhaps equally important, this type of pressure rise should be more uniformly distributed around the ring, which is not the case at RHIC. Therefore, this mechanism can be ruled out.

3. Beam loss - electron desorption induced pressure instability. Compared with the slow moving ions, electrons are accelerated and hit the chamber wall by each bunch passing. Abundant electrons can be available once the electron multipacting takes place, which is happening routinely at RHIC. Electron cloud induced pressure rise usually tends to approach a saturation, due to the space charge limitation of electron cloud. However, positive ions created by beam loss and/or beam gas ionization may reduce this space charge force, and hence to cause pressure rise beyond the limit. Two aspects related to this possibility will be discussed in this note. 1 . Sources and patterns of the ions' production. 2. The run-away pressure rises observation at RHIC for run 2003 and run 2004, for reality check.

Many issues and questions associated with this problem are relevant in terms of better understanding of the RHIC pressure rise. These issues and the related parameters will be discussed, and questions will be presented. 


\section{Ion desorption induced pressure instability}

The mechanism of the ion desorption induced pressure instability can be described as follows. Ions are generated by the beam gas ionization, then being pushed to the wall by the beam potential. At low beam intensity, this contribution to the pressure rise will be absorbed by the vacuum pumping, and an equilibrium pressure will be reached. As the beam intensity increases, there will be a threshold that pumping cannot handle the newly generated gas molecules, then the pressure instability will take place. The mechanism can be shown by the following equation [2, and references therein],

$$
P=\frac{P_{0} S}{S-\eta_{i} \sigma_{i} N_{i} f_{0}}
$$

where the pressure $P$ is in Torr, $P_{0}$ is the initial pressure, the effective pumping speed $S$ in $\ell s^{-1} m^{-1}$ has included effects of the pipe conductance and the distance between pumps, etc. The ionization cross section $\sigma_{i}$ is in $m^{2}$, where the residual gas of $N_{2}$ or $C O$ equivalent is used (the pumping speed is also $N_{2}$ or $C O$ equivalent). $\eta_{i}$ is the ion desorption rate, $N_{i}$ is the total beam ions in the ring, and $f_{0}$ is the revolution frequency.

The singularity of the equation (1) indicates possible pressure instability. The following criterion shows the instability condition in terms of the ion desorption rate,

$$
\eta_{i} \geq \frac{S}{\sigma_{i} N_{i} f_{0}}
$$

Unlike the electrons, which hit the wall after each bunch's kick, the ions will receive kicks of many bunches before reaching the wall. The energy the ion gained from the passing bunches can be calculated by [2- 4],

$$
\langle\Delta E\rangle=\frac{e}{2 m_{p}}\left(\frac{e K N_{b h} Z_{0}}{2 \pi b}\right)^{2} \ln \left(\frac{b}{a}\right)
$$

where the proton with the mass of $m_{p}$ is taken as an example. The chamber and beam radii are $b$ and $a$, respectively, $Z_{0}$ is the vacuum impedance, and there are $K$ bunches with bunch intensity of $N_{b h}$ contribute to the ions' energy for the ions to reach the chamber wall. To determine $K$, a simple program is written. The ions are assumed to be generated at the vicinity of the beam, i.e. at $a$ from the center of the chamber. Acceleration follows by continuous kicks of the beam bunches. Once the travel distance of $b-a$ is 
completed, the ions' lifetime and the bunch number $K$ can be obtained. The energy of the ions reaching the wall, $\langle\Delta E\rangle$, is thus calculated.

Take $b=6.1 \mathrm{~cm}$ and $a=4.7 \mathrm{~mm}$ at the RHIC warm region, and assume 110 bunches with $10^{9}$ gold ions per bunch. The ions' lifetime, number of bunches that contributed, and the ions' energy reaching the wall are listed in Table 1, where the corresponding parameters of ISR and LHC are listed for comparison.

\begin{tabular}{c|cccc} 
& RHIC & ISR & LHC & \\
& $A u^{79+}$ & $p$ & $p$ & \\
\hline$E_{k}$ & 8.9 & 26 & 7,000 & $\mathrm{GeV} / \mathrm{u}$ \\
$T_{0}$ & 12.8 & 3.14 & 88.6 & $\mu s$ \\
$N_{b h}$ & $79 \times 10^{9}$ & $10^{14}$ & $1.1 \times 10^{11}$ & Charge \\
$M$ & 120 & 1 & 3,560 & \\
$b, h / v$ & 61 & $80 / 30$ & $22 / 18$ & $\mathrm{~mm}$ \\
$a, h / v$ & 4.7 & $22 / 5$ & 0.28 & $\mathrm{~mm}$ \\
$\tau, h / v$ & 2.5 & $0.52 / 0.20$ & $0.34 / 0.28$ & $\mu s$ \\
$K, h / v$ & 23.5 & $0.17 / 0.06$ & $13.4 / 11.1$ & \\
$\langle\Delta E\rangle, h / v$ & 10.5 & $252 / 344$ & $87.2 / 85.2$ & $\mathrm{eV}$
\end{tabular}

Table 1

In Table $1, E_{k}$ is the beam's kinetic energy, $T_{0}$ is the revolution time, $M$ is the bunch number filling the ring, $\tau$ is the ions' lifetime, $K$ is the number of the bunches passed before the ions hit the wall, and $\langle\Delta E\rangle$ is the ions' energy reaching the wall.

For ISR ion desorption induced vacuum instability, the desorption rate of $\eta_{i}=4$ was used to explain the ISR vacuum pressure run-away $[3,5,6]$. This desorption rate is consistent with the ions' energy when hitting the wall, higher than $300 \mathrm{eV}$ as shown in Table 1, together with the unbaked steel surface, see Table 3 in [2]. For the baked chambers and increased pumping speed, the ISR later was able to increase the beam intensity by more than 10 times, to $1.2 \times 10^{15}$ protons, at $60 \mathrm{~A}$ of beam current.

The desorption rate for the ions hitting at RHIC chamber wall, $10.5 \mathrm{eV}$, is comparable with the mean energy of secondary ions released from ionization and sputtering, which is about $10 \mathrm{eV}$ [7]. It is also known that the threshold energy of sputtering yield, to overcome the binding energy in the target, is from $20 \mathrm{eV}$ to $50 \mathrm{eV}[7,8]$. Above this threshold, the ion desorption rate rapidly increases from $10^{-5}$ to 0.1 of the desorption rate at $300 \mathrm{eV}$, in an 
energy increment of $50 \mathrm{eV}$ [8]. In other words, if we take the ion desorption rate at $300 \mathrm{eV}$ as 3, and the threshold energy as $20 \mathrm{eV}$, then the desorption rate is roughly $10^{-5}$ at $20 \mathrm{eV}$, and it is less than 0.3 at $70 \mathrm{eV}$. Therefore, the ions pushed to the wall by the RHIC beam is probably harmless, and the chance that has caused run-away pressure rise is small at the best.

\section{Beam-loss induced pressure instability}

Assume that the beam loss is caused by the pressure rise due to the mechanism of stripping, capture, nuclear and atom collisions, or combined. If the lost ions desorb enough gas which surpass the pumping capacity, then pressure instability may take place. The equation (1) can be used to describe the situation by redefining some variables. The beam gas ionization cross section $\sigma_{i}$ is replaced by $\sigma$, which is now for the relevant cross section of stripping, capture, nuclear and atom collision, or combined. The desorption rate $\eta_{i}$ is replaced by $\eta$, which is now for the high energy beam ions, rather than the low energy ions with less than a few hundred $e V$. The ion desorption rate for the low energy ions is somewhat well known, but the one of the high energy ions is not. One important factor is that the high energy ions are usually lost at grazing angles, rather than the perpendicular angle in normal incidents. Given shallow scraping angles and the rough target surface, the sputtering effect is not straightforward to describe quantitatively.

Since the gold ions at the RHIC are fully stripped, only the capture, nuclear and atomic collision cross sections might be relevant. It was calculated [9] that at the RHIC injection, the capture cross section is $\sigma_{c}=2.5 \times 10^{-29}$ $\mathrm{m}^{2}$. On the other hand, the nuclear collision cross section is about an order larger than the capture cross section, which is $\sigma_{n}=4.7 \times 10^{-28} \mathrm{~m}^{2}$.

Take the conditions considered for the RHIC situation, i.e., pumping speed $S=13 \ell s^{-1} m^{-1}, 110$ bunches with $10^{9}$ gold ions per bunch in the ring. Using the revised equation (2), similarly to the revision of the equation (1), and taking $\sigma_{n}=4.7 \times 10^{-28} \mathrm{~m}^{2}$, it can be shown the desorption rate needs to be $3.3 \times 10^{9}$ to reach the threshold of vacuum instability.

As comparison, pressure instability might have been observed at GSI SIS18. There were $10^{9} U^{28}$ ions in the ring, and the revolution frequency was $200 \mathrm{kHz}$ at $10 \mathrm{MeV} / \mathrm{u}$. The stripping cross section is about $\sigma_{s}=10^{-20} \mathrm{~m}^{2}$. Taking the effective pumping speed of $S=20 \mathrm{ls}^{-1} \mathrm{~m}^{-1}$, then the desorption rate of $10^{4}$ is sufficient for the pressure instability. Considering the beam ions' 
scraping on the wall, this desorption rate is smaller than the ones reported at several machines. The desorption rate of $A u^{31}$ at $1 \mathrm{MeV} / u$ was measured as $10^{5}$ at the AGS Booster [10]. The one of $P b^{53}$ at LEAR was estimated as $10^{5}$ [11]. In an experiment at the CERN LINAC3, the desorption rate of $2 \times 10^{4}$ was measured for the $4 \mathrm{MeV} / u \mathrm{~Pb}^{54}$ ions with a not very shallow incident angle of $14 \mathrm{mrad}$ [12].

Because of the large beam loss, the vacuum instability may not be presented as the run-away pressure rise. For example, in the case of the pressure rise observed at the GSI SIS 18 , the beam injected as $7 \times 10^{9} U^{28}$ ions, which is reduced to less than $10^{9} U^{28}$ ions in 0.3 second, and the pressure rise was initially observed, but it started to drop immediately as the beam intensity reduced below $10^{9} U^{28}$ ions.

Equally important factor in this mechanism is that the pressure rise should be uniformly distributed around the ring, since the beam will be lost at downstream due to the pressure rise, then the pressure rise will propagate longitudinally in the ring. In contrast with the GSI, the RHIC pressure run-away pressure rise happened locally.

\section{Beam-loss electron-multipacting induced pres- sure instability}

\subsection{Relevant parameters}

To look into the beam loss related electron multipacting, parameters relevant to the RHIC pressure rise are presented below. These parameters provide a basis to speculate possible mechanism, and it is also useful for the reality check.

1. For RHIC gold beam with $10^{9}$ ions per bunch, the bunch charge is $N_{b h}=7.9 \times 10^{10}$. The bunch length is $6 \mathrm{~m}$ at the injection, therefore, the bunch charge line density is $1.32 \times 10^{10} \mathrm{~m}^{-1}$.

2. With the neutralization factor of $\zeta_{e}=0.01$, electron line density is $\lambda_{e}=1.32 \times 10^{8} \mathrm{~m}^{-1}$. The neutralization factor 0.01 is taken from general observations of the electron cloud. It is roughly agreeable with the observations in usual electron cloud. The electron density for higher intensity beam could lead to higher neutralizations. 
3. Assuming half of the 110 bunches having developed electron cloud, with the revolution frequency $f_{0}=78 \mathrm{kHz}$, and the electron desorption rate of $\sigma_{e}=0.1$, the electron cloud with the line density of $\lambda_{e}=1.32 \times 10^{8}$ $m^{-1}$ generated molecules are $\lambda_{e} f_{0} \times 110 / 2 \times \sigma_{e}=5.66 \times 10^{13} \mathrm{~s}^{-1} \mathrm{~m}^{-1}$. Given the effective pumping speed at RHIC as $S=13 \mathrm{ls}^{-1} \mathrm{~m}^{-1}$, the pressure rise caused by the electron cloud is $5.66 \times 10^{13} /\left(k_{m} S\right)=1.3 \times$ $10^{-7}$ Torr, where $k_{m}=3.3 \times 10^{22} \mathrm{~m}^{-3}$ is the number of molecules in $1 \mathrm{~m}^{3}$ at 1 Torr. This level of pressure rise is often observed at RHIC for electron cloud induced pressure rise.

4. If the average kinetic energy of the secondary electrons equals the potential energy of electrons at the beam pipe, then these electrons cannot survive due to space charge. A rough but usable estimate is to equate the two to get the saturated electron line density [4]

$$
\lambda_{e, s a t}=\frac{3 \pi E_{s e}}{e Z_{0} c}
$$

where if the average energy of the secondary electrons is taken as $E_{s e}=2.5 \mathrm{eV}$, then the saturated line density is $\lambda_{e, s a t}=1.3 \times 10^{9}$ $m^{-1}$. Compared with the beam line density $\lambda=1.32 \times 10^{10} \mathrm{~m}^{-1}$, the neutralization factor is $\zeta_{e, s a t}=0.1$, which leads to pressure rise of $1.3 \times 10^{-6}$ Torr. Note that this saturation limit can be affected, maybe significantly, by the positive ions. The possible sources are described in the following 3 aspects.

5. The first source of the non-beam ions is the beam gas ionization. Take the gold ionization cross section of $79 \times 10^{-22} \mathrm{~m}^{2}$ per charge (might be a little too large [13]), and the average beam current $I=0.11$ $A$, which is from 110 bunches of gold ion with $10^{9}$ ions per bunch. At $P=1.3 \times 10^{-7}$ Torr, the beam gas ionization created ions are $k_{m} P \sigma I / e=3.3 \times 10^{22} \times 1.3 \times 10^{-7} \times 79 \times 10^{-22} \times 0.11 / e=2.3 \times 10^{13}$ $s^{-1} m^{-1}$. Since that the average lifetime of ions is $2.5 \mu s$ at RHIC, the ionization produced ions are $\lambda_{i, I O N}=2.3 \times 10^{13} \times 2.5 \times 10^{-6}=5.8 \times 10^{7}$ $m^{-1}$ at $P=1.3 \times 10^{-7}$ Torr.

6. The second source of the non-beam ions is the cloud electron ionization. The ionization cross section of electrons is peaked at around $100 \mathrm{eV}[1$, and references therein]. Given the average energy of cloud electrons for RHIC gold beam, $35 \mathrm{eV}$ [2], the cloud electron ionization cross section 
can be taken as $1.2 \times 10^{-20} \mathrm{~m}^{2}$, the same as [14]. The electron path is $2 \times 0.06 \times 110 / 2 \times f_{0}=515 \times 10^{3} \mathrm{~ms}^{-1}$, where the radius of the pipe is $0.06 \mathrm{~m}$, and half of the bunches is assumed to have developed electron cloud. Given the electron line density of $\lambda_{e}=1.32 \times 10^{8} \mathrm{~m}^{-1}$, which is associated with the pressure rise of $1.3 \times 10^{-7} \mathrm{Torr}$, then the ions generated from the cloud electrons are $1.32 \times 10^{8} \mathrm{~m}^{-1} \times 1.2 \times 10^{-20} \mathrm{~m}^{2} \times$ $515 \times 10^{3} \mathrm{~ms}^{-1} \times 3.3 \times 10^{22} \mathrm{~m}^{-3} \times 1.3 \times 10^{-7}=3.5 \times 10^{9} \mathrm{~s}^{-1} \mathrm{~m}^{-1}$. Since that the average lifetime of ions is $2.5 \mu \mathrm{s}$, the cloud electron produced ions are $\lambda_{i, E C}=3.5 \times 10^{9} \times 2.5 \times 10^{-6}=8.8 \times 10^{3} \mathrm{~m}^{-1}$ at $P=1.3 \times 10^{-7}$ Torr.

7. The third source of the non-beam ions is from the beam loss. A halo type beam loss with the very shallow angle may have high desorption rate, such as $10^{7}$, which is sometimes observed at RHIC [15]. Using this desorption rate, assuming $10^{6}$ ions lost per second per meter, then in $2.5 \mu \mathrm{s}$ there is $2.5 \times 10^{7} \mathrm{~m}^{-1}$ ions generated. Let the loss to take place in $10 \mathrm{~m}$ along the ring, then for total 110 bunches with $10^{9}$ ions per bunch, the loss rate is less than $0.01 \%$ per second, which is not inconsistent with the machine observations. Another factor is that in general the ions' production is smaller than the molecules' production in the sputtering. Nevertheless, given the high changed $A u^{79}$ ions, the ratio of the ions and molecules might be higher. Here we assume that ratio is unity.

\subsection{Possible mechanism of run-away type pressure rise}

Among three possible sources of ions, the cloud electron looks most unlikely to be a dominant one. At the modest pressure rise of $P=1.3 \times 10^{-7}$ Torr, the cloud electron generated ion line density is $\lambda_{i, E C}=8.8 \times 10^{3} \mathrm{~m}^{-1}$, it is only $0.015 \%$ of that from the beam gas ionization for the same pressure rise. It is also only $0.033 \%$ of that from the beam loss. Hence we focus on the other two factors.

Since the ion production of beam loss is not depending on the pressure, it is more pronounced at lower pressure, with the less beam gas ionizations. Using the parameters given in 7 in last subsection, the ions may fully neutralize the electron line density of $\lambda_{e}=2.7 \times 10^{7} \mathrm{~m}^{-1}$, which is corresponding to the pressure rise of $P=2.7 \times 10^{-8}$ Torr. This implies that given a beam scraping scenario, it is possible that the beam loss generated ions help for 
the electron multipacting with extending the electron's lifetime by non-trivial neutralization.

On the other hand, the contribution of the beam gas ionization cannot be overlooked. Firstly, at the pressure of $P \geq 10^{-7}$ Torr, this mechanism may provide significant number of ions to extend the secondary electron's lifetime, and hence to cause electron multipacting. Secondly, at high pressure rise, this mechanism can raise the electron cloud space charge limitation, and hence to cause run-away type pressure rise.

Two legitimate questions are,

1. What is the role of the beam gas ionization at low pressure rise? Can this mechanism along affect the electron multipacting threshold?

2. How are these two mechanisms involved in the run-away type pressure rise? Which one is more important?

To answer the first question, observation of the RHIC electron cloud induced pressure rise in past three runs is a key. From the beginning, the correlation of the static pressure with the pressure rise threshold was one of major focuses in the investigation. No solid correlation was found, and therefore, no suggestion to fix the static pressure as high priority was made. The upper level of the static pressure in the ring, however, is below $P=10^{-8}$ Torr. It is possible that at higher static pressure, the picture will change and the correlation will show up. It would be interesting to see if the static pressure at $P \geq 10^{-7}$ Torr would affect the threshold of electron multipacting. On the other hand, strong electron multipacting is frequently observed for the locations with the static pressure of $P \leq 10^{-11}$ Torr. Therefore, it seems unlikely that the beam gas ionization along is a dominant mechanism.

To answer the second question, a study of the run-away type pressure rises in run 2003 and run 2004 might help. In Table 2, total 7 run away type pressure rise during the run 2003 and run 2004 are shown. 


\begin{tabular}{|c|c|c|c|c|}
\hline Fill & $\begin{array}{c}\text { Intensity } \\
10^{9} A u\end{array}$ & Bunch mode & $\begin{array}{c}\text { Pressure rise } \\
\text { Torr }\end{array}$ & Location \\
\hline 2818, Run-3 & 72 & 110 & $2 \times 10^{-5}$ & Yi2 \\
\hline 3107, Run-3 & 82 & 110 & $2 \times 10^{-3}$ & Yo1 \\
\hline 4124, Run-4 & 40 & 56 & $10^{-3}$ & Yo4 \\
\hline 4313, Run-4 & 60 & 61 & $4 \times 10^{-4}$ & Bi8 \\
\hline 4316, Run-4 & 49 & 61 & $5 \times 10^{-4}$ & Yo4 \\
\hline 4372, Run-4 & 56 & 61 & $3 \times 10^{-4}$ & Bi8 \\
\hline 4413, Run-4 & 54 & 56 & $1.2 \times 10^{-5}$ & $\mathrm{Bi} 8$ \\
\hline
\end{tabular}

Table 2

These pressure rises are shown in Figure 1, where one may notice that the run-away type pressure rises are started from anywhere from $10^{-10} \mathrm{Torr}$ to $10^{-6}$ Torr , depending on the previous pressure rise history.

Several comments are due,

1. It can be noticed that these run-away pressure rises have very different growth rates. Moreover, the growth rates are not dependent on the pressure started, indicating that the beam gas ionization is not a dominant mechanism. It is interesting to notice that, however, at the pressure $>5 \times 10^{-5}$ Torr, the growth rates become very similar. The vacuum pumps have ceased to work at this level of pressure. It is possible that the beam gas ionization starts to take dominant effect at this high pressure.

2. The irregular pattern of the pressure rises, compared with the more uniform type of ISR ion desorption induced run-away pressure rise, may indicate that the ion desorption of ISR type is not a dominant mechanism in RHIC, consistent with the RHIC parameter discussed in the section 3 .

3. The beam intensities are well below $110 \times 10^{9}$ gold ions, only $40 \times$ $10^{9}$ gold ions in 4124, making it more difficult to speculate for ion desorption caused run-away pressure rise.

4. All the cases were with the significant beam loss along with the pressure rise at the relevant locations. As examples, in Figure 2, the pressure rise of 4124 is compared with the beam loss monitor data at the location, Yo4. And in Figure 3, the pressure rise of 4372 is compared with the pin diodes data at the location, Bi8. 


\subsection{Discussion}

The RHIC run-away type pressure rise is probably not from a single mechanism. The beam gas ionization is unlikely a dominant mechanism. On the other hand, the beam gas ionization created ions, which extend the secondary electrons' lifetime and reduce the space charge, is likely taking effect, at least at high pressure rise. The beam loss has two effects. One is that it produces molecules, which directly contribute to the pressure rise. Another is that it produces ions. Electron desorption from the cloud electrons is the most likely contributor for the pressure rise, due to its abundance, activity, and its productive energy hitting the wall. Once the space charge limit removed, it is reasonable to speculate that electrons could cause run-away type pressure rise at $\mathrm{RHIC}$.

A few questions need to be raised.

1. In addition to the space charge limit, the electron density has another limit, which is the neutralization factor. In our calculation, the neutralization factor of 1 implies the pressure rise to $1.3 \times 10^{-5}$ Torr, which is higher than $5 \times 10^{-6}$ Torr, where the vacuum pumps cease to work. To get to this limit, numerous positive ions will be in the pipe, which are supposed to invite the electrons to stay, awaiting the kick from the passing bunches. A model is needed for a better vision of this scenario.

2. So far the run-away type pressure rise has mostly been observed for gold beams. One may suggest that due to high charge state, fully stripped gold ions produce more ions than other species with lower charge state. Again, more data and a model is needed here to get a quantitative estimate.

3. In a more complete picture, at least three mechanism should be included, these are 1. Beam gas ionization with ions' production. 2. Beam loss with the productions of both molecules and ions. 3. Effect of active electrons, perhaps with the production not only the molecules, but also the ions. 


\section{References}

1. W. Fischer, U. Iriso, and E. Mustafin, C-AD/AP 173, Dec. 2004.

2. S.Y. Zhang, C-AD/AP 67, Jan. 2002.

3. O. Grobner, EPAC 98, p.3589, Stockholm, Sweden,1998

4. L. Vos, LHC Project Note 150, CERN, July 1998.

5. I.R. Collins, et al, Int. Workshop on Performance Improvement of $e^{+} e^{-}$ Factories, KEK, Japan, Sep. 1999.

6. O. Grobner, CERN 99-05, p.127, 1999.

7. Sputtering by Particle Bombardment III, Edited by R. Behrisch and K. Wittmaack, Spriger-Verlag, New York, 1991.

8. R.V. Stuart and G.K. Wehner, J. Appl. Phys. 33, 2345, 1962.

9. M.J. Rhoades-Brown and M. Harrison, AD/RHIC-106, Dec. 1991

10. S.Y. Zhang and L.A. Ahrens, PAC99, p.3294, New York, 1999.

11. J. Hansen, et al. LHC/VAC Note, 2001-007, CERN, July, 2001.

12. E. Mahner, J. Hansen, J.M. Laurent, and N. Madsen, Phys. Rev. STAccel. Beams 6, 013201, 2003.

13. B. Franzke, CERN 92-01, p.100, 1992.

14. O. Grobner, CARE-HHH Workshop, CERN, Nov. 2004.

15. S.Y. Zhang, 'Ion desorption at RHIC', HB-2004, Bensheim, Germany, Oct. 2004. 


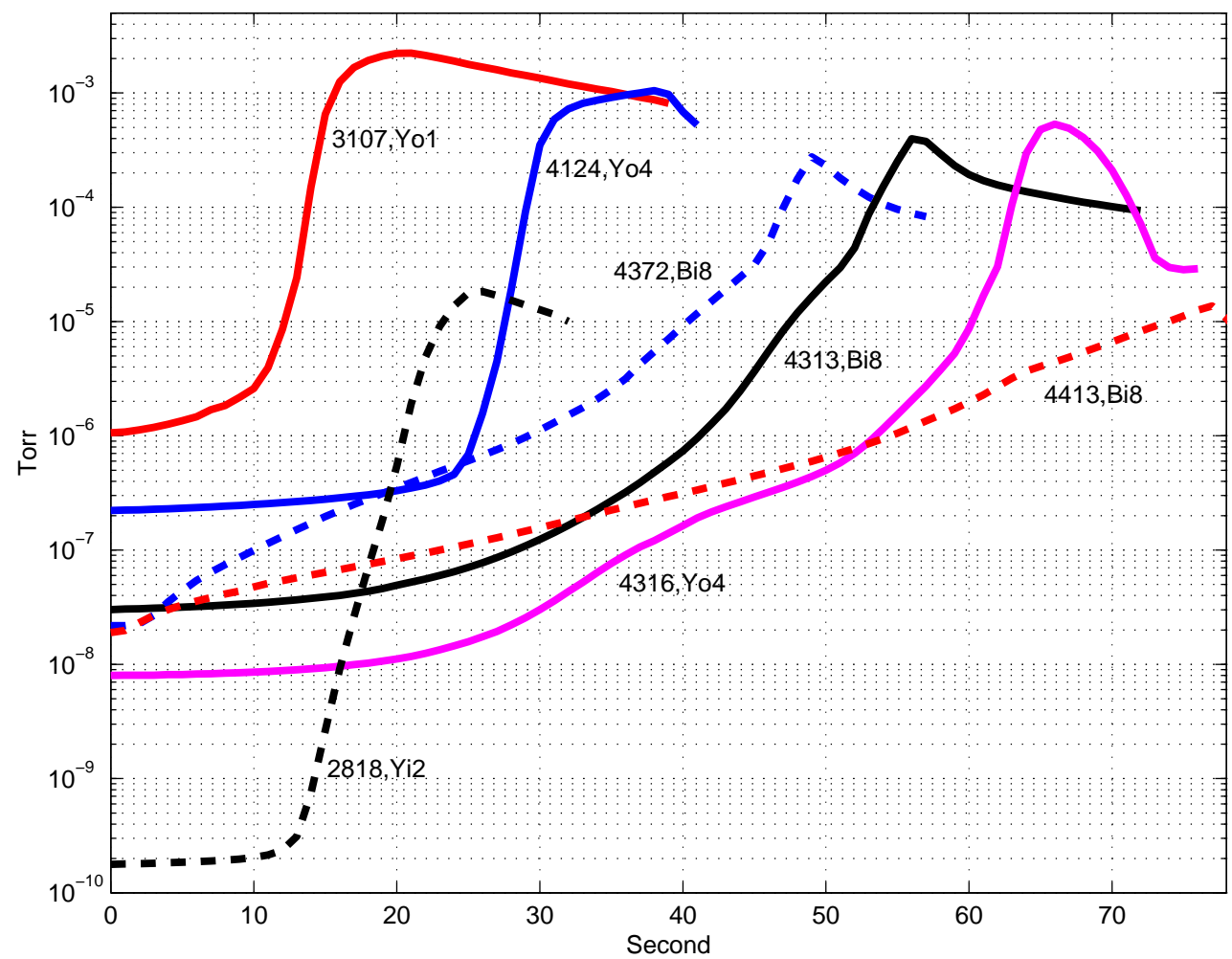

Figure 1: Run-away type pressure rise in run 2003 and run 2004. All cases are for gold beams. The cases of 2818 and 3107 are in run 2003, at Q3-Q4 single beam straight section Yo1 and Yi2. Rest are in run 2004. Pressure rises of fills 4124 and 4316 are at Yo4. Pressure rises of fills 4313, 4316, and 4413 are at Bi8, the blue collimation section. All the locations were unbaked. 

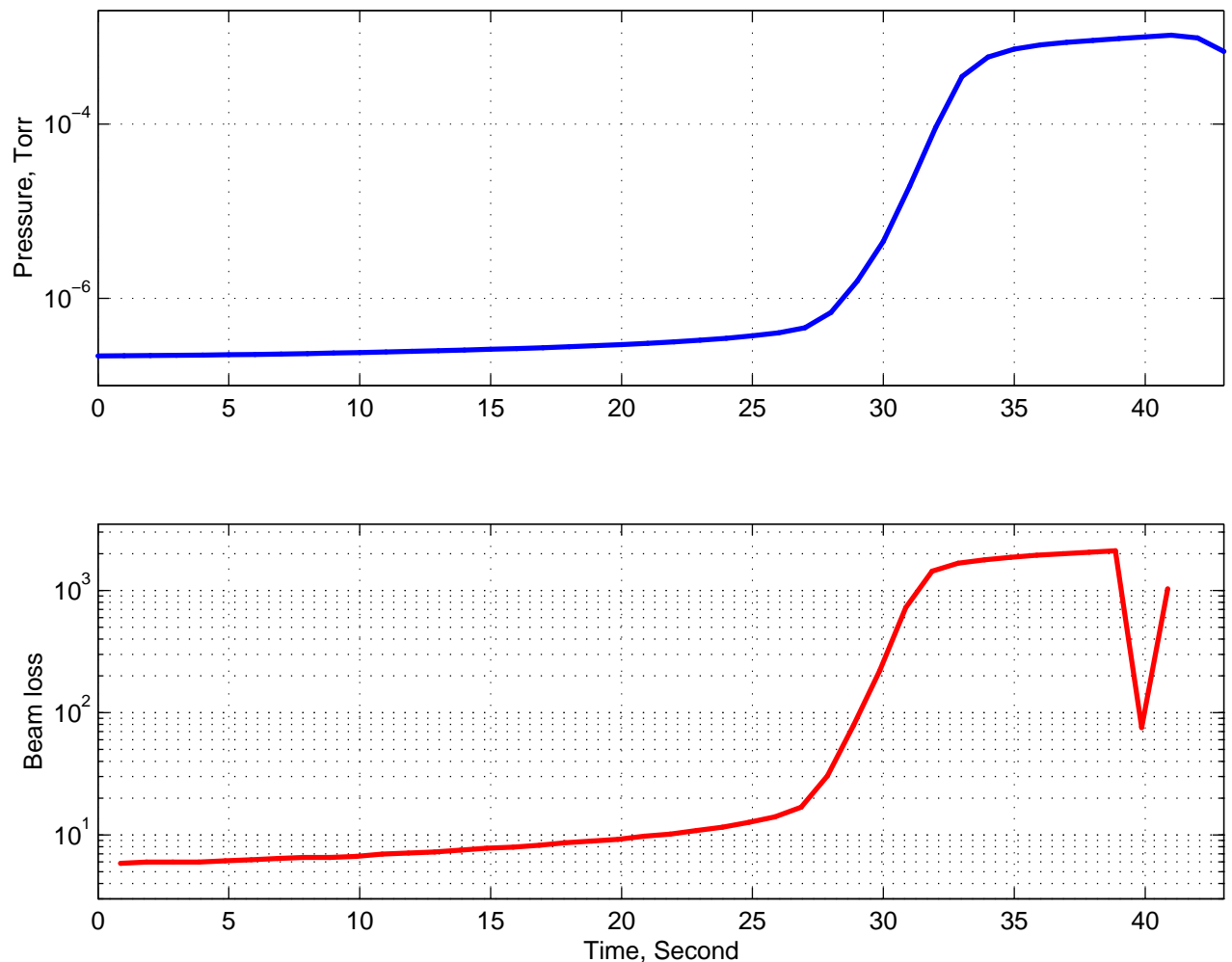

Figure 2: Pressure rise of 4124 at Yo4 and the beam loss monitor data at the location 

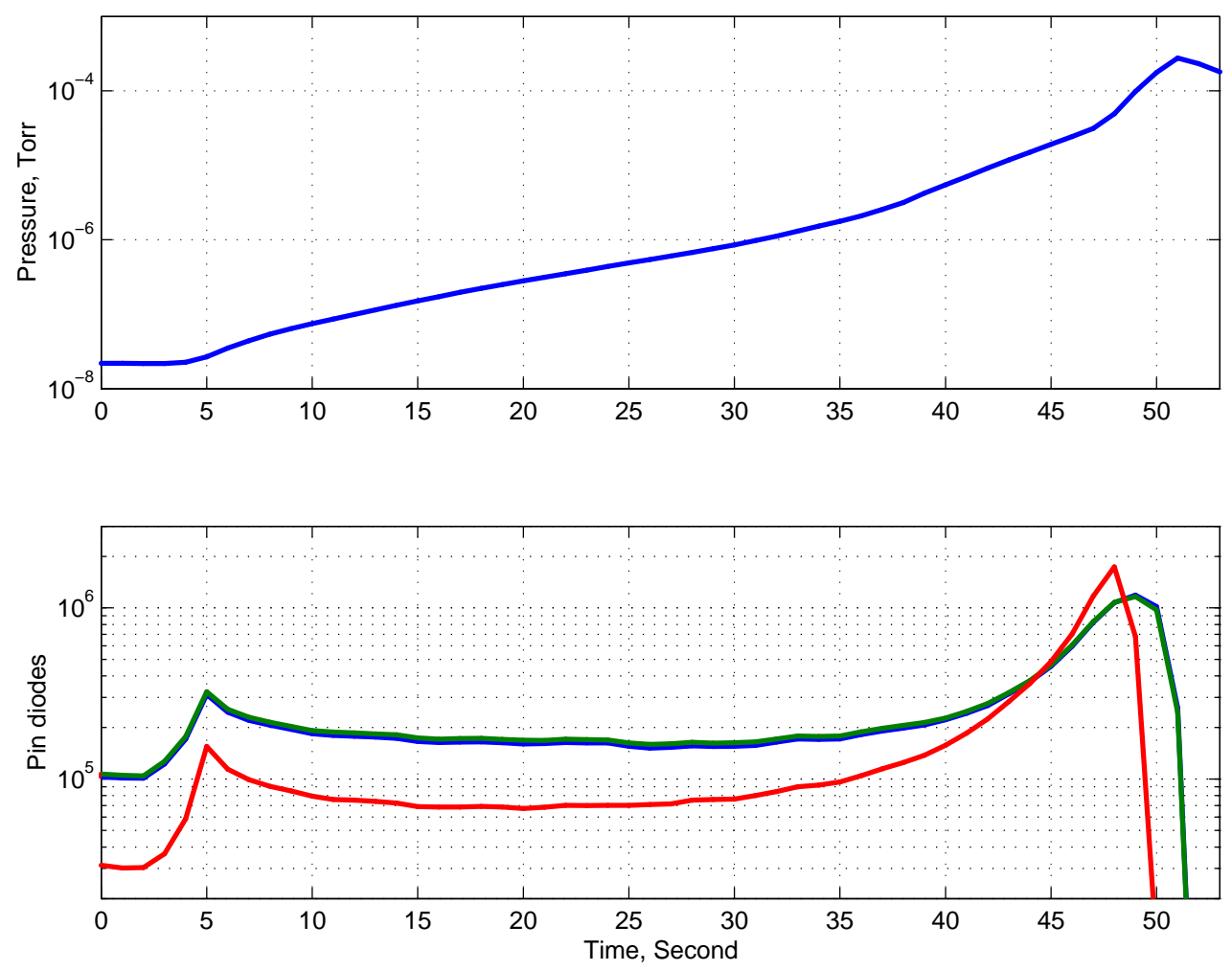

Figure 3: Pressure rise of 4372 at Bi8, and the three sets of pin diodes data at the location. 